\title{
Current techniques of prostate biopsy: an update from past to present
}

\author{
Mohamed Essam Noureldin ${ }^{1,2,3}$, Martin J. Connor ${ }^{1}$, Nicholas Boxall ${ }^{4}$, Saiful Miah ${ }^{4}$, Taimur Shah ${ }^{1,2}$, \\ Jochen Walz
}

${ }^{1}$ Imperial Prostate, Division of Surgery, Department of Surgery and Cancer, Imperial College London, London, UK; ${ }^{2}$ Department of Urology, Charing Cross Hospital, Imperial College Healthcare NHS Trust, London, UK; ${ }^{3}$ Department of Urology, Ain Shams University Hospitals, Cairo, Egypt; ${ }^{4}$ Department of Urology, Cambridge University Hospitals, Cambridge, UK; ${ }^{5}$ Department of Urology, Institut Paoli-Calmettes Cancer Centre, Marseille, France

Contributions: (I) Conception and design: S Miah, ME Noureldin, MJ Connor; (II) Administrative support: MJ Connor; (III) Provision of study materials or patients: ME Noureldin; (IV) Collection and assembly of data: ME Noureldin, MJ Connor, J Walz; (V) Data analysis and interpretation: ME Noureldin; (VI) Manuscript writing: All authors; (VII) Final approval of manuscript: All authors.

Correspondence to: Mr Mohamed Essam Noureldin. Imperial Prostate, Department of Surgery and Cancer, Imperial College London, Charing Cross Campus, Fulham Palace Road, London, W6 8RF, UK. Email: mohamed.noureldin@nhs.net.

\begin{abstract}
Knowing the demerits of the transrectal ultrasound guided biopsy has led to a shift in the general techniques and technology of the prostate biopsy. In addition, the advances in magnetic resonance imaging (MRI) sensitivity and the evidence from the PROMIS trial about its ability to detect clinically significant tumours encourage urologists and industry to implement MRI-guided targeted biopsy. However, the science is still looking how to improve safety and increase its cancer detection rate (CDR).
\end{abstract}

Keywords: Prostate cancer; prostate neoplasm; biopsy; magnetic resonance imaging (MRI); grade

Submitted Aug 19, 2019. Accepted for publication Sep 04, 2019.

doi: $10.21037 /$ tau.2019.09.20

View this article at: http://dx.doi.org/10.21037/tau.2019.09.20

\section{Introduction}

Prostate biopsy has undergone a recent diagnostic revolution driven by technological advances in magnetic resonance imaging (MRI) and standardised image reporting systems (i.e., PI-RADS). This, crucially, has been underpinned by high-level clinical trial evidence $(1,2)$. For over two decades the urological community made little progress beyond the use of conventional transrectal ultrasound guided prostate biopsy (TRUS-GB).

However, Ahmed and colleagues' landmark PROMIS trial demonstrated the limited accuracy of conventional TRUS-GB in detecting clinically significant prostate cancer (csPCa), whilst simultaneously validating the benefits of pre-biopsy multi-parametric MRI (mpMRI). Following this, the PRECISION randomised controlled study confirmed the accuracy of multiparametric MRI (mpMRI) targeted prostate biopsy by improving detection of clinically significant (Gleason $\geq 3+4$ ) and reduced clinically nonsignificant prostate cancer detection rates (CDRs) when compared to TRUS-GB alone.

Such landmark studies have led to fervent academic interest in the utility of novel image-fusion technology, the role of transperineal prostate biopsy and in image-based prostate cancer diagnostic pathway design (Figure 1).

Our review aims to provide an update on current techniques utilized in prostate biopsy with respect to prebiopsy imaging, image-fusion and anatomical approach. In addition, we set to identify outstanding research questions and registered clinical trials in this rapidly evolving field.

\section{Conventional TRUS-GB}

TRUS-GB was first described by Hodge and colleagues' in 1989 (1). The group reported on a novel image-guided biopsy utilizing a spring-loaded gun to target any ultrasound 


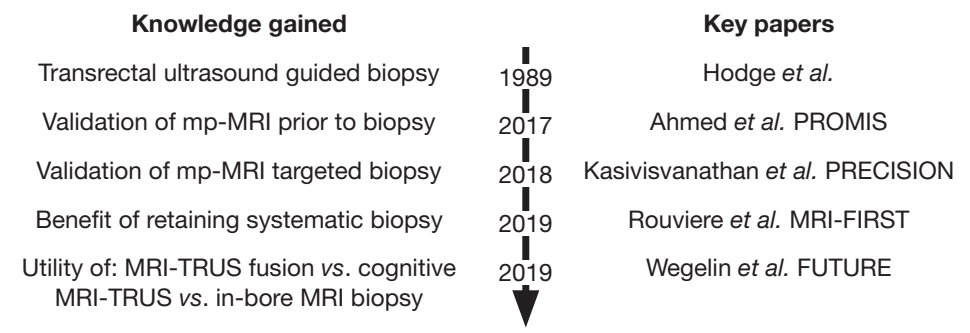

Figure 1 Evolution of prostate biopsy knowledge following introduction of transrectal ultrasound guided biopsy.

visible lesion (i.e., hypoechoic defects). In 136 consecutive men with an abnormal digital rectal examination (DRE), a 6 core, $1.5 \mathrm{~cm}$ TRUS-GB produced a CDR of $62 \%$. This was a significant improvement in terms of cancer detection on previously available techniques but cannot be compared to current definitions off csPCa.

The number of cores increased to 10 or 12 cores to become standard of care $(3,4)$. Clinical diagnostic pathways were developed using PSA or abnormal DRE as a triage tool to decide on TRUS-GB. Such pathways led to an overdetection of lower grade and non-significant tumours which lead to downstream increases in radical treatment rates (2). Jin and colleagues' further undermined the value of TRUSGB by reporting that there was a $40 \%$ upgraded in final Gleason score on whole-mount prostatectomy histology $(3,5,6)$.

\section{Template mapping prostate biopsy (TPM)}

Although TRUS-GB offered a reasonable way to sample the prostate gland, there was still a reported underdiagnosis of $25-30 \%$ of clinically significant tumours (7). This could happen due to under-sampling of the lesions or missing the lesions completely which was proved on the repeated biopsy studies (1).

One of the solutions for that was to increase the numbers of cores and to shift to the perineal route to get better access to the anterior part of the prostate. As a standard protocol it was done under general anaesthesia which enables taking a large number of cores. In the CDR most of the studies supported the idea that there is an improved CDR in comparison to the TRUS-GB (8).

Although Jones and colleagues' argued that such a saturated biopsy strategies did not yield improved CDRs in biopsy naïve men and increased their risk of post-biopsy complications (9). Other well-documented limitations were that such biopsy necessitated a general anaesthesia with all the inherent risks related to this. In addition, the risk of urinary retention was very high and a proportion of patients required a urethral catheter post-procedure (10). More importantly, overall improved cancer detection led to a rise in the non-significant disease (11).

Template mapping biopsy is no longer recommended in the updated NICE guidelines [2019] in the diagnosis of biopsy naïve men unless in a clinical trial setting. However, TPM has persisted as a useful tool in focal (ablative) therapy clinical trials where interval sampling of the outof-field disease status over time is of increasing importance in predicting men who will progress over time (12). Such a strategy has been coined "TPM-Ablate-TPM".

\section{Utility of pre-biopsy prostate mpMRI}

The PROMIS trial demonstrated the clinical utility of mpMRI for men with suspected prostate cancer prior to biopsy (2). The authors' reported the positive predictive value (PPV) of mpMRI as $90 \%$ compares to $51 \%$ conventional TRUS-GB without pre-biopsy imaging [OR 8.2; 95\% CI: 4.7-14.3; $\mathrm{P}<0.0001]$ (1).

Similarly, Drost and colleagues' systematic review reported that mpMRI could detect $90 \%$ of clinically significant prostate cancers (13). In addition, systematic prostate biopsy was reported to miss approximately $37 \%$ of the significant lesions.

Such evidence has lead the NICE updating its guidelines, which now recommend pre-biopsy MRI pathway and subsequent biopsy for any lesion identified as more than Likert 3 or if PSA density is above $0.15 \mathrm{ng} / \mathrm{mL}$ or PSA velocity greater than $0.75 \mathrm{ng} /$ year (14).

\section{Validation of mpMRI targeted prostate biopsy}

The concept of systematic sweeping of the prostate to detect the cancer has been undermined by the strength of 
evidence supporting targeting suspected lesions or regions of interest (ROI) on the pre-biopsy mpMRI.

Panebianco and colleagues' RCT randomised 1,140 men to conventional systematic TRUS-GB or mpMRI targeted and systematic TRUS biopsy. The cancer detection in the MRI targeted biopsy group was compared to the histology of subsequent whole-mount prostatectomy sample (15). It was proven that the MRI targeted biopsy was superior to the TRUS in the CDR as the MRI targeted group showed detection rate of $94 \%$ while the random TRUS biopsy had $89 \%$ detection rate. In addition, there was no clinically significant cancer detected in those with a negative MRI and negative first biopsy. These outcomes reinforce the role of mpMRI targeted biopsy in initial prostate cancer diagnosis.

Such an mpMRI targeted biopsy approach has gained popularity following the publication of PRECISION which emphasized that mpMRI targeted biopsy, in comparison to standard TRUS biopsy, resulted in improved clinically significant cancer detection ( $38 \%$ vs. 26\%). In addition, the lower detection of the non-significant cancers in the targeted group $(9 \%)$ compared to $(22 \%)$ in the TRUS group. The limitations of this trial were that conventional systematic biopsy was omitted in the mp-MRI targeted arm (5).

Schoots and his colleagues' systematic review comparing the mpMRI targeted biopsy to the systematic biopsy stated that CDR of clinically significant tumours was slightly higher in the targeted biopsy side (sensitivity $0.91,95 \%$ CI: $0.87-0.94$ vs. $0.76,95 \%$ CI: 0.64-0.84). However due to the lack of comparison with the histology after radical prostatectomy it was recommended to combine the both techniques together (16).

The PICTURE trial was a blinded randomized trial including 249 patients who underwent MRI-targeted biopsy and template TP biopsy blinded to the MRI results. This trial showed that the CDR of clinically-significant tumours was $40 \%$ in the targeted group, while it was only $17 \%$ in the TP group and they recommended targeted biopsy to be used as a standard of care (17).

Also Radtke et al. (18) compared the saturation biopsy to the MRI-targeted biopsy in 294 patients that had MRItargeted biopsy and then saturation TP biopsy (average of 24 cores) then the highest Gleason score was compared in each group. The TP biopsy missed about $20 \%$ of the clinically significant tumours while the targeted biopsy missed $12 \%$ of them. There was a slight superiority in Gleason 7 detection in the targeted group. They concluded at the end that the gold standard of cancer detection in primary biopsy should be a combination of systematic and targeted biopsy. Abraham et al. studied the detection rates in 1,837 men retrospectively in the repeated biopsy setting. This study recommended that MRI targeted, or imagebased biopsy should be used in the repeat biopsy setting rather than the systematic biopsy (19).

\section{Biopsy anatomical approach: trans-rectal or trans-perineal?}

\section{Procedure considerations}

Anatomical approach is an important consideration in prostate biopsy, with widespread practice of both transrectal and transperineal approaches. Transrectal prostate biopsy is usually an office procedure where the patient lies in the lateral decubitus position. A DRE is performed prior to the procedure to detect any nodularity. The ultrasound probe should be inserted slowly and with pressure to dilatate the anal sphincter. A prostate block is then applied by injecting local anaesthetic in the space between the prostate capsule and the seminal vesicles (20). Measuring the prostate size is recommended prior to infiltration of local anaesthesia. A 12-core sextant biopsies include sampling the peripheral zone bilaterally and the transitional zone in the base, mid and apex of the prostate (Figure 2) (21).

On the other hand, transperineal biopsy is performed in the lithotomy position using the brachytherapy grid and stepper to fix the probe (Figure 3) (22).

Typically about 22 cores are taken from 14 to 18 location using the Barzell map to cover the prostate (23). This technique optimizes access to the anterior lesions, especially in the big glands. However, there is a higher risk of urinary retention that should be considered with the template biopsy as per the PICTURE study (10).

\section{Biopsy related toxicity}

The popularity of systematic transperineal biopsy (TPB) has increased with the publication of extremely favourable sepsis rates $(<1.0 \%)(24,25)$. However, TRUS biopsy remains the most common procedure to diagnose prostate cancer. TRUS is associated with higher relative complications such as life-threatening sepsis, urinary retention and bleeding. A large European study reported the sepsis rate post TRUS at $4.2 \%$. In addition, an adverse predictor of sepsis was an increased number of cores. 

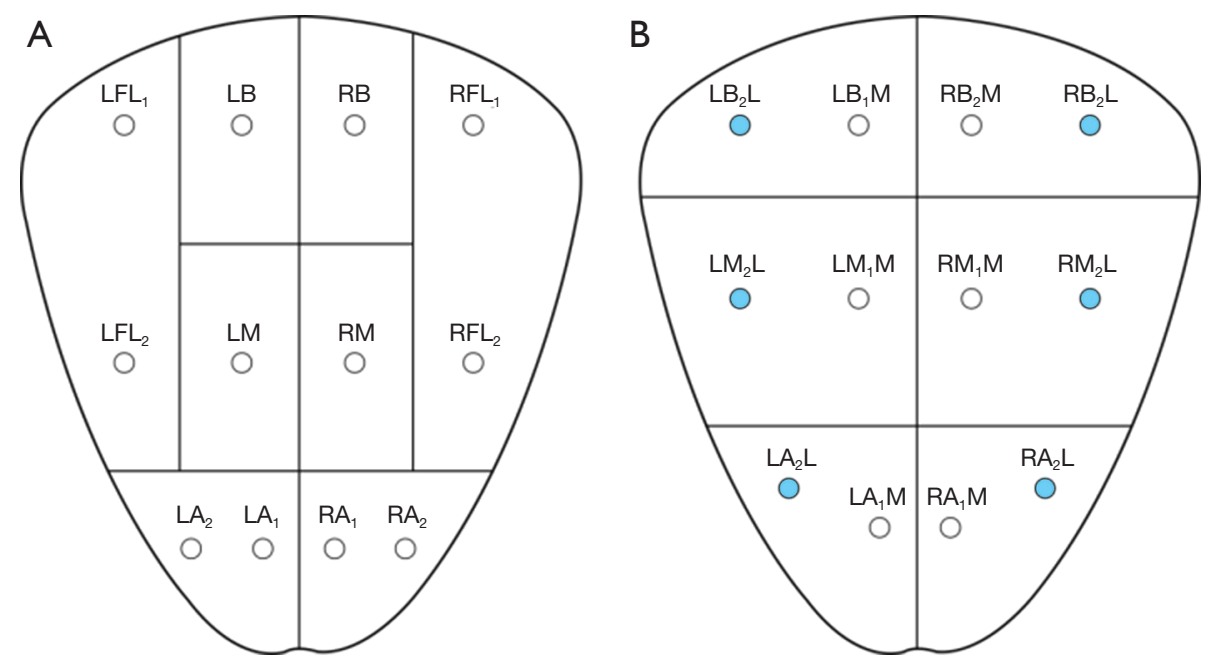

Figure 2 Sextant cores of the TRUS-GB. Total of either 8 (A) or 6 (B) containers. Inking the lateral core in each container can provide additional information regarding orientation (B). TRUS-GB, transrectal ultrasound guided prostate biopsy; LFL, left far lateral; RFL, right far lateral; LB, left base; RB, right base; LM, left mid; RM, right mid; LA, left apex; RA, right apex; LBL, left base lateral; RBL, right base lateral; LML, left mid lateral; RML, right mid lateral; LMM, left mid medial; RMM, right mid medial; LAL, left apex lateral; RAL, right apex lateral; LAM, left apex medial; RAM, right apex medial.

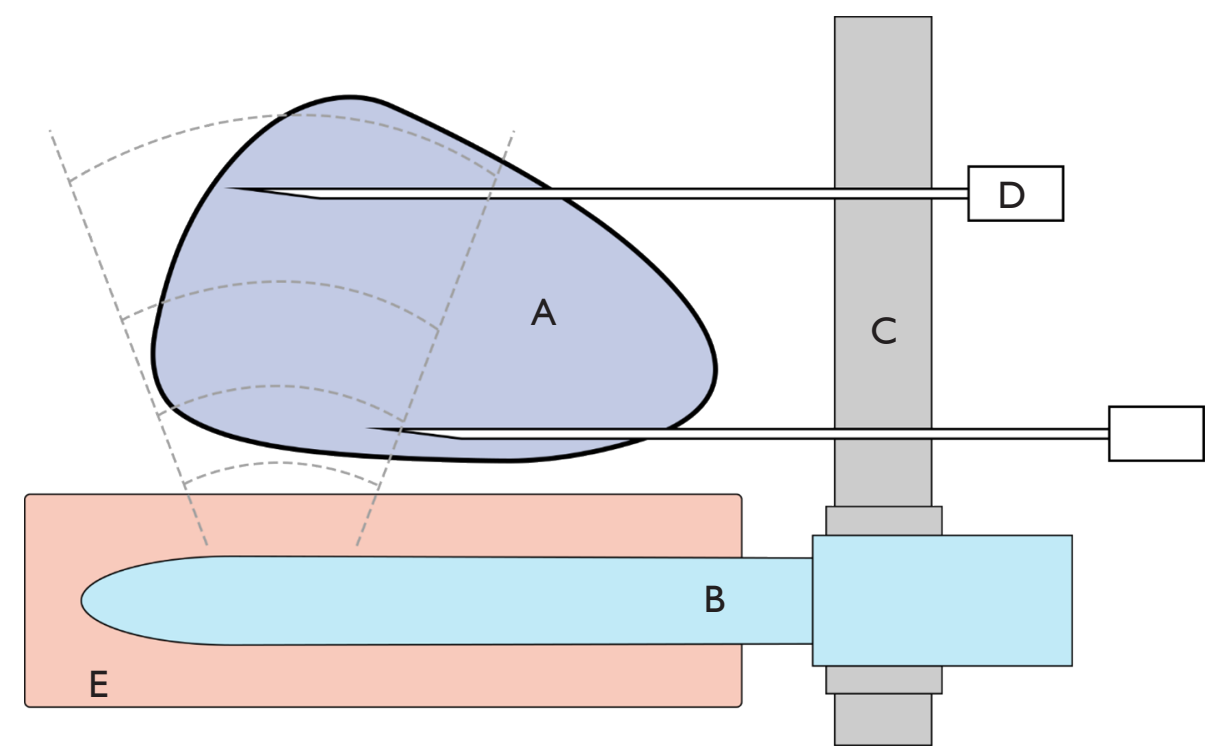

Figure 3 Transperineal prostate biopsy. (A) Prostate; (B) transrectal ultrasound probe; (C) grid; (D) biopsy needle; (E) rectum.

\section{Learning curve and sampling considerations}

TRUS biopsy remains attractive as it can be performed in a clinic setting under local anaesthesia with a short learning curve in comparison to the TP biopsy which usually requires in most of the cases sedation or general anaesthesia (26).

In addition to that it was mainly operator dependent and there was a difficulty in reaching the anterior lesion tumours which will lead to a higher rate of missing the significant cancers in the anterior regions (27).

Recently, using a deep prostate block, TP biopsy was offered under local anaesthesia. In the last few years multiple studies compared between the TP targeted biopsy 
Table 1 Definition of image fusion technique

\begin{tabular}{llll}
\hline Image fusion technique & Definition & Reported benefits & Reported negatives \\
\hline $\begin{array}{l}\text { Cognitive (visually } \\
\text { estimated) fusion }\end{array}$ & $\begin{array}{l}\text { The operator forms a visual link between the MRI images } \\
\text { and the real time ultrasound pictures then targeting the } \\
\text { suspected areas }\end{array}$ & Low cost & Very operator-dependent \\
Software assisted fusion & $\begin{array}{l}\text { The MRI images are uploaded and registered to the real } \\
\text { time ultrasound by a software. Using this combined } \\
\text { picture, a target area requiring biopsy is projected }\end{array}$ & High costs & $\begin{array}{l}\text { Moderately operator } \\
\text { dependent }\end{array}$ \\
& $\begin{array}{l}\text { Fusion between prior MRI images and a real time MRI } \\
\text { during the procedure }\end{array}$ & $\begin{array}{l}\text { Specific material for MR } \\
\text { environment, cost and } \\
\text { time intensive }\end{array}$ & Little operator dependent \\
\hline
\end{tabular}

under local with the other techniques of the biopsies. DiBianco et al. stated in his 244 patients underwent TP biopsy under local that it was safe technique and feasible for most patients. This technique was also supported by Bass et al. who tested local TP biopsy over 181 men and the outcomes showed the feasibility of the technique with high CDRs (28). To avoid the brachytherapy stepper discomfort to the patients and the complicated settings of the TP biopsy, free hand techniques were introduced also recently for local settings and it showed a competitive CDR and safety $(29,30)$.

\section{Understanding image-fusion techniques}

There are three main techniques for the targeted biopsy: (I) cognitive fusion; (II) Software assisted fusion; (III) in-bore biopsy (16) (Table 1).

The answer of which is the best question is still debatable as every technique has a different learning curve, cost and variation in its CDR in the literature. Reviewing the literature will show the uncertainty in choosing the ideal technique.

In the PICTURE trial, 249 patients underwent template prostate mapping biopsies with cognitive and software fusion targeted biopsy. They were compared in the CDR of the clinically significant tumours. Half of the $17 \%$ of the clinically significant tumours detected by the template were in the non-targeted areas. Cognitive biopsies detected significant cancer in $31 \%$ of the patients and the softwareassisted biopsies showed $28 \%$ of them. The missed significant cancers were 23 cases in the cognitive group, and they were detected by the software-assisted technique. On the other hand, the software missed 18 cases detected by the cognitive. The study concluded that MRI-targeted biopsy is sensitive in detecting clinically significant tumours but the detection rate was maximized by combining cognitive and software-assisted fusion (17).

The SmartTarget Biopsy Trial enrolled 141 men with previous biopsy and patients underwent randomized cognitive and software-assisted biopsies. Both techniques combined detected 93 cases of clinically significant tumours (72\%). Each technique missed 13 cases which were detected by the other modality. So, it concluded that a combination of both techniques gives the highest detection rate of the clinically significant tumours (31).

Arsov et al. compared the MRI guided in-bore biopsy to systematic biopsy in about 200 patients over 3 years prospectively and randomized (32). The results of the histology between the groups were compared regarding the CDR and the rate of clinically significant tumours. CDR in the in-bore group was $37 \%$ while it was $39 \%$ in the systematic group. More clinically significant tumours were detected in the systematic group (32\% vs. 29\%). The study could not prove that in-bore targeted biopsy alone showed any superiority over systematic biopsy.

The FUTURE trial also demonstrated the efficacy of the MRI targeted biopsy over the systematic sampling of the prostate: 642 patients who had a negative biopsy before were included in the study due to still having the concern of prostate cancer as their PSA was equal or more than 4 or abnormal DRE. They all underwent MRI. Two hundred and thirty-one of them had equal or more than PIRADS 3 lesions so they had targeted MRI with additional systematic biopsies. Significant cancers were detected in $34 \%$ of the patients with targeted biopsy and systematic biopsy detected only $16 \%$ of them. Moreover, the targeted biopsy showed significant cancers in $21 \%$ of patients who had negative systematic biopsy and that strengthened the targeted biopsy 
even in patients who had previous biopsy (33).

In a recent systematic review including about 43 studies comparing the in-bore, software fusion biopsy and cognitive biopsy. The review concluded that although the overall cancer detection was higher in the fusion biopsy in comparison to the cognitive, they both have similar rates in detecting the significant lesions (34).

\section{Outstanding research questions}

One of the questions that still have unclear answer is the role of the systematic samples in addition to targeted samples.

In one of the multicentre study (35) which included about 800 patients who had TP systematic biopsy and more than half of the patients had additional targeted biopsies. The results suggest that using each technique of them alone will lead to fewer of the Gleason $3+4$ and $4+3$ and missing part of lesions in comparison to combing both.

However, in a recent systematic review by Schoots et al. (16), it was evidenced from the reviewed literature that it would be concerning if it concluded that the targeted biopsy alone could detect cancer more than the systematic according to the data he found. It recommended comparing the results of each technique to the histology results post-prostatectomy which is still a missing part in the literature.

In a recent meta-analysis including 68 studies and 8 RCTs, 14,709 patients had MRI targeted biopsy and systematic biopsy, the MRI targeted biopsy detected more clinically significant cancers in men than the systematic template biopsy with 1.16 detection ratio (95\% confidence interval) and this concluded that the MRI targeted biopsy is an attractive alternative to the systematic biopsy (35).

It is evidenced now that the mpMRI has changed the field of prostate cancer diagnosis. However, there is still a big challenge to get expert radiologists available to report those cancer patients besides that the learning curve for accurate report on PI-RADS or Likert score is almost challenging as the biopsy procedure itself. One of the big challenges for the future is to get a good radiomics standardized which may help in one day to make a complete module of automated report by the machine.

\section{Conclusions}

The prostate biopsy evolved in recent years. The aim of all these changes is to reach the ideal biopsy technique which has low morbidity, a high sensitivity in cancer detection with minimal cost and optimize the patient experience.

TRUS biopsy without prior mpMRI leads to overdetection of clinically non-significant disease and underdetection of clinically significant disease. MRI-targeted PB is now evolving as the gold standard approach. Uncertainty exists regarding the role of systematic prostate biopsy and the optimal image-fusion technique.

\section{Acknowledgments}

Funding: MJC is funded by the Wellcome trust.

\section{Footnote}

Provenance and Peer Review: This article was commissioned by the Guest Editors (Martin J. Connor, Saiful Miah, Taimur T. Shah, Hashim U. Ahmed) for the series "Prostate Imaging and Focal Therapy" published in Translational Andrology and Urology. The article was sent for external peer review organized by the Guest Editors and the editorial office.

Conflicts of Interest: All authors have completed the ICMJE uniform disclosure form (available at http://dx.doi. org/10.21037/tau.2019.09.20). The series "Prostate Imaging and Focal Therapy" was commissioned by the editorial office without any funding or sponsorship. MJC, SM and TS served as the unpaid Guest Editors of the series. The authors have no other conflicts of interest to declare.

Ethical Statement: The authors are accountable for all aspects of the work in ensuring that questions related to the accuracy or integrity of any part of the work are appropriately investigated and resolved.

Open Access Statement: This is an Open Access article distributed in accordance with the Creative Commons Attribution-NonCommercial-NoDerivs 4.0 International License (CC BY-NC-ND 4.0), which permits the noncommercial replication and distribution of the article with the strict proviso that no changes or edits are made and the original work is properly cited (including links to both the formal publication through the relevant DOI and the license). See: https://creativecommons.org/licenses/by-nc-nd/4.0/. 


\section{References}

1. Kasivisvanathan V, Rannikko AS, Borghi M, et al. MRITargeted or Standard Biopsy for Prostate-Cancer Diagnosis. N Engl J Med 2018;378:1767-77.

2. Ahmed HU, El-Shater Bosaily A, Brown LC, et al. Diagnostic accuracy of multi-parametric MRI and TRUS biopsy in prostate cancer (PROMIS): a paired validating confirmatory study. Lancet 2017;389:815-22.

3. Sruogis A, Jankevicius F, Mickys U. Prostatic biopsy technique. Historical review. Medicina 2005;41:957-67.

4. Baco E, Rud E, Eri LM, et al. A randomized controlled trial to assess and compare the outcomes of two-core prostate biopsy guided by fused magnetic resonance and transrectal ultrasound images and traditional 12-core systematic biopsy. Eur Urol 2016;69:149-56.

5. Giganti F, Moore CM. A critical comparison of techniques for MRI-targeted biopsy of the prostate. In: Transl Androl Urol 2017;6:432-43.

6. Jin BS, Kang SH, Kim DY, et al. Pathological upgrading in prostate cancer patients eligible for active surveillance: Does prostate-specific antigen density matter? Korean J Urol 2015;56:624-9.

7. Siddiqui MM, Rais-Bahrami S, Turkbey B, et al. Comparison of MR/Ultrasound Fusion-Guided Biopsy with Ultrasound-Guided Biopsy for the Diagnosis of Prostate CancerMR/Ultrasound Fusion Biopsy for Prostate CancerMR/Ultrasound Fusion Biopsy for Prostate Cancer. JAMA 2015;313:390-7.

8. Eichler K, Hempel S, Wilby J, et al. Diagnostic value of systematic biopsy methods in the investigation of prostate cancer: a systematic review. J Urol 2006;175:1605-12.

9. Jones JS, Patel A, Schoenfield L, et al. Saturation technique does not improve cancer detection as an initial prostate biopsy strategy. J Urol 2006;175:485-8.

10. Miah S, Eldred-Evans D, Simmons LAM, et al. Patient reported outcome measures for transperineal template prostate mapping biopsies in the PICTURE study. J Urol 2018;200:1235-40.

11. Taira AV, Merrick GS, Galbreath RW. Performance of transperineal template-guided mapping biopsy in detecting prostate cancer in the initial and repeat biopsy setting. Prostate Cancer Prostatic Dis 2010;13:71-7.

12. Dickinson L, Ahmed HU, Kirkham AP, et al. A multicentre prospective development study evaluating focal therapy using high intensity focused ultrasound for localised prostate cancer: the INDEX study. Contemp Clin Trials 2013;36:68-80.
13. Drost FH, Osses DF, Nieboer D, et al. Prostate MRI, with or without MRI-targeted biopsy, and systematic biopsy for detecting prostate cancer. Cochrane Database Syst Rev 2019;4:CD012663.

14. Graham J, Kirkbride P, Cann K, et al. Prostate cancer: summary of updated NICE guidance. BMJ 2014;348:f7524.

15. Panebianco V, Barchetti F, Sciarra A, et al. Multiparametric magnetic resonance imaging vs. standard care in men being evaluated for prostate cancer: A randomized study. Urol Oncol 2015;33:17.e1-17.e7.

16. Schoots IG, Roobol MJ, Nieboer D, et al. Magnetic Resonance Imaging-targeted Biopsy May Enhance the Diagnostic Accuracy of Significant Prostate Cancer Detection Compared to Standard Transrectal Ultrasoundguided Biopsy: A Systematic Review and Meta-analysis. Eur Urol 2015;68:438-50.

17. Simmons LA, Kanthabalan A, Arya M, et al. Accuracy of Transperineal Targeted Prostate Biopsies, Visual Estimation and Image Fusion in Men Needing Repeat Biopsy in the PICTURE Trial. J Urol 2018;200:1227-34.

18. Radtke JP, Kuru TH, Boxler S, et al. Comparative Analysis of Transperineal Template Saturation Prostate Biopsy Versus Magnetic Resonance Imaging Targeted Biopsy with Magnetic Resonance Imaging-Ultrasound Fusion Guidance. J Urol 2015;193:87-94.

19. Abraham NE, Mendhiratta N, Taneja SS. Patterns of repeat prostate biopsy in contemporary clinical practice. J Urol 2015;193:1178-84.

20. Li M, Wang Z, Li H, et al. Local anesthesia for transrectal ultrasound-guided biopsy of the prostate: A meta-analysis. Sci Rep 2017;7:40421.

21. Mohammed W, Davis NF, Elamin S, et al. Six-core versus twelve-core prostate biopsy: a retrospective study comparing accuracy, oncological outcomes and safety. Irish J Med SCI 2016;185:219-23.

22. Pal RP, Elmussareh M, Chanawani M, et al. The role of a standardized 36 core template-assisted transperineal prostate biopsy technique in patients with previously negative transrectal ultrasonography-guided prostate biopsies. BJU Int 2012;109:367-71.

23. Valerio M, Anele C, Charman SC, et al. Transperineal template prostate-mapping biopsies: an evaluation of different protocols in the detection of clinically significant prostate cancer. BJU Int 2016;118:384-90.

24. Guo LH, Wu R, Xu HX, et al. Comparison between ultrasound guided transperineal and transrectal prostate biopsy: a prospective, randomized, and controlled trial. Sci 
Rep 2015;5:16089.

25. Loeb S, Vellekoop A, Ahmed HU, et al. Systematic review of complications of prostate biopsy. Eur Urol 2013;64:876-92.

26. Chang DTS, Challacombe B, Lawrentschuk N. Transperineal biopsy of the prostate-is this the future? Nat Rev Urol 2013;10:690.

27. Abdelsayed GA, Danial T, Kaswick JA, et al. Tumors of the anterior prostate: implications for diagnosis and treatment. Urology 2015;85:1224-8.

28. Bass EJ, Donaldson IA, Freeman A, et al. Magnetic resonance imaging targeted transperineal prostate biopsy: a local anaesthetic approach. Prostate Cancer Prostatic Dis 2017;20:311-7.

29. DiBianco JM, Mullins JK, Allaway M. Ultrasound guided, freehand transperineal prostate biopsy: an alternative to the transrectal approach. Urology Practice 2016;3:134-40.

30. Lian H, Zhuang J, Wang W, et al. Assessment of freehand transperineal targeted prostate biopsy using multiparametric magnetic resonance imaging-transrectal ultrasound fusion in Chinese men with prior negative biopsy and elevated prostate-specific antigen. BMC Urol 2017;17:52.

31. Hamid S, Donaldson IA, Hu Y, et al. The SmartTarget Biopsy Trial: A Prospective, Within-person Randomised,

Cite this article as: Noureldin ME, Connor MJ, Boxall N, Miah S, Shah T, Walz J. Current techniques of prostate biopsy: an update from past to present. Transl Androl Urol 2020;9(3):1510-1517. doi:10.21037/tau.2019.09.20
Blinded Trial Comparing the Accuracy of Visualregistration and Magnetic Resonance Imaging/Ultrasound Image-fusion Targeted Biopsies for Prostate Cancer Risk Stratification. Eur Urol 2019;75:733-40.

32. Arsov C, Rabenalt R, Blondin D, et al. Prospective Randomized Trial Comparing Magnetic Resonance Imaging (MRI)-guided In-bore Biopsy to MRI-ultrasound Fusion and Transrectal Ultrasound-guided Prostate Biopsy in Patients with Prior Negative Biopsies. Eur Urol 2015;68:713-20.

33. Exterkate L, Wegelin O, Van Melick H, et al. 624 - The FUTURE trial: A RCT on MRI targeted prostate biopsy. Comparison of targeted and systematic biopsy outcomes. European Urology Supplements 2018;17:e896-e897.

34. Wegelin O, van Melick HHE, Hooft L, et al. Comparing three different techniques for magnetic resonance imaging-targeted prostate biopsies: a systematic review of in-bore versus magnetic resonance imaging-transrectal ultrasound fusion versus cognitive registration. Is there a preferred technique? Eur Urol 2017;71:517-31.

35. Hansen NL, Barrett T, Kesch C, et al. Multicentre evaluation of magnetic resonance imaging supported transperineal prostate biopsy in biopsy-naïve men with suspicion of prostate cancer. BJU Int 2018;122:40-9. 